\title{
Morphology and Mechanical Properties of Low Density Polyethylene/Multi-walled Carbon Nanotubes Nanocomposites
}

\author{
Lin-jian SHANGgUAN ${ }^{1}$, Fang HUANG ${ }^{2 *}$
}

${ }^{1}$ School of Mechanical Engineering, North China University of Water Conservancy and Electric Power. Collaborative
Innovation Center of Water Resources Efficient Utilization and Guarantee Engineering, Zhengzhou, 450011, China
${ }^{2}$ College of Material Science and Engineering, Henan University of Technology, Zhengzhou, 450001, China

cross ${ }^{\text {ref }}$ http://dx.doi.org/10.5755/j01.ms.23.2.15074

Received 29 May 2016; accepted 08 July 2016

\begin{abstract}
Low density polyethylene/multi-walled carbon nanotubes (LDPE/MWNTs) nanocomposites were manufactured with screw extrusion and injection moulding. The morphology and mechanical properties of LDPE/MWNTs nanocomposites were investigated. The results show that, at low content of MWNTs, they could uniformly disperse into LDPE and contribute to the crystallization of LDPE; LDPE crystal maintains the original structure in the nanocomposites; compared to LDPE, the increases in tensile strength and Young's modulus for LDPE/3.0 wt.\% MWNTs nanocomposite can be up to $27 \%$ and $148 \%$, respectively.

Keywords: LDPE/MWNTs nanocomposites, morphology, mechanical properties.
\end{abstract}

\section{INTRODUCTION}

In comparison with pure polymer, due to light weight, flexibility, high electrical conductivity, strength, easy processing, and exceptional physical and mechanical properties of carbon nanotubes (CNTs), polymer/CNTs nanocomposites have drawn more wide attention [1-5]. A variety of CNTs/polymer nanocomposites and films have been widely used in the design of new, super-strong composites in academia and industry.

In most cases, single wall CNTs (SWNTs) and multiwall CNTs (MWNTs), are often applied to fabrication of nanocomposite. In comparison with MWNTs, SWNTs exhibit poor enhancements in a polymer matrix, probably due to bundle formation [6]. Therefore, MWNTs are generally preferred to SWNTs.

Low-density polyethylene (LDPE) is one of polyethylene varieties and allowed to be indirect contact with food by FDA (the Food and Drug Administration) [7]. LDPE can be easily processed, uses cheaply, and has high toughness, and so on [8]. Although there is a lot of literature of LDPE/MWNTs nanocomposite [9-12], they focus on the preparation of the nanocomposite.

In this work, to utilize and enhance the mechanical properties of LDPE, LDPE/MWNTs nanocomposites were manufactured with screw extrusion and injection moulding. The effects of MWNTs on the morphology, crystallization and crystal structure of LDPE, tensile strength and Young's modulus of LDPE/MWNTs nanocomposites were investigated. This kind of nanocomposites is expected to broaden the application of LDPE.

\footnotetext{
* Corresponding author. Tel.: +86-13513896470.

E-mail address: huang_polymer@163.com (F. Huang)
}

\section{EXPERIMENTAL DETAILS}

\subsection{Materials}

LDPE (DJ200, by free radical high-pressure polymerization) was purchased from Shanghai Petrochemical (China) by free radical high-pressure polymerization. Its density and melt index are $0.918 \mathrm{~g} \mathrm{~cm}^{-3}$

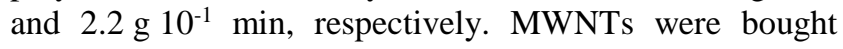
from Shenzhen Nanotech Port. PEG (Aldrich, $\mathrm{Mn}=10000)$ was chosen as a dispersant and plasticizer.

3-(trimethoxysilyl) propyl methacrylate (KH-570) and paraffin wax (chemical grade) were chosen as a coupling agent and lubricant, respectively.

\subsection{Preparation}

MWNTs, KH-570 and PEG were put into ethanol; the mixture was sonicated for $30 \mathrm{~min}$, then dried in a vacuum box. The mixtures of LDPE, treated MWNTs and 0.1 wt.\% of paraffin wax were mixed with co-rotating twin-screw extruder. The amount of MWNTs addition were 0.5, 1.0, 2.0 and $3.0 \mathrm{wt} . \%$, respectively. The extruded composites dried in a vacuum box at $100{ }^{\circ} \mathrm{C}$ for $6 \mathrm{~h}$. The composites were molded by an injection machine with a screw diameter in $33.0 \mathrm{~mm}$ and L/D ratio of 20 .

\subsection{Test methods}

Scanning electron microscope (SEM, JSM-5900LV) was used to observe the fractured surface of LDPE/MWNTs nanocomposites. Transmission electron microscopy (TEM, Hitachi H-800, $200 \mathrm{kV}$ accelerating voltage) was used for examining MWNTs. The thin sections of LDPE/MWNTs nanocomposites (about 50$70 \mathrm{~nm}$ thickness) were prepared by a Leica EM FC6 ultramicrotome. A differential scanning calorimeter (DSC, Du Pont 9900) was measured the thermal properties of the nanocomposites. The crystallinity $(X)$ of LDPE was 
calculated from the following relation:

$X=\Delta H_{m} / \Delta H_{m}^{0}$,

where $\Delta H_{m}$ and $\Delta H_{m}^{0}\left(69.55 \mathrm{cal} \mathrm{g}^{-1}\right.$ [13]) are the melting enthalpy of sample and $100 \%$ crystallizing polyethylene, respectively.

Wide-angle X-ray diffraction (WAXD) patterns were tested via a D8 Advance X-ray diffractometer (Bruker, Germany). WAXD spectra was obtained over the $2 \theta$ range of $5-60^{\circ}$. The tensile properties of LDPE/MWNTs nanocomposite were tested by the Instron testing machine (model 4302).
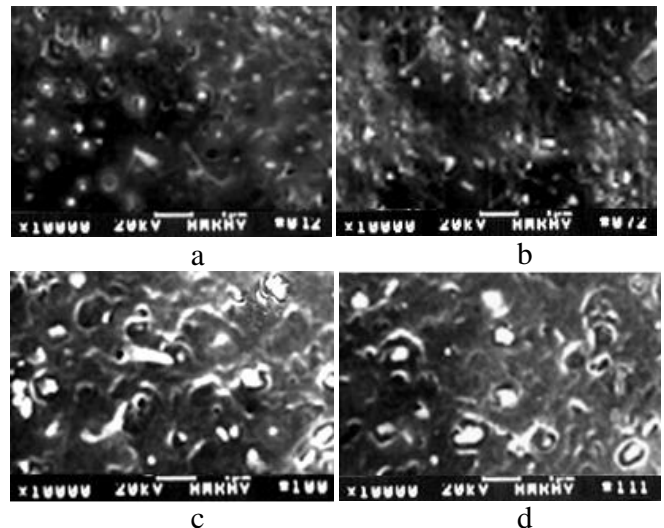

Fig. 1. SEM of LDPE/MWNTs: a - LDPE/0.5 wt.\% MWNTs; $\mathrm{b}$-LDPE $/ 1.0 \quad \mathrm{wt} \% \quad$ MWNTs; $\quad \mathrm{c}$-LDPE/2.0 wt.\% MWNTs, d-LDPE/3.0 wt.\% MWNTs

\section{RESULTS AND DISCUSSION}

The enhancement of nano-fillers in polymer-based nanocomposites is mainly determined by their dispersion in polymer matrices. Therefore, it is necessary to study morphological characterization of MWNTs in polymer. The dispersion state of MWNTs in LDPE/MWNTs nanocomposites was observed with SEM. Fig. 1 shows SEM images of MWNTs in LDPE/MWNTs nanocomposites with $0.5,1.0,2.0$ and $3.0 \mathrm{wt} . \%$ MWNTs content, respectively; the selected SEM observations are LDPE/MWNTs nanocomposites cryo-fractured surfaces.

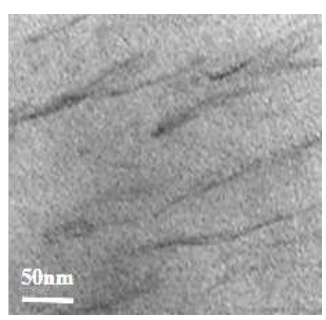

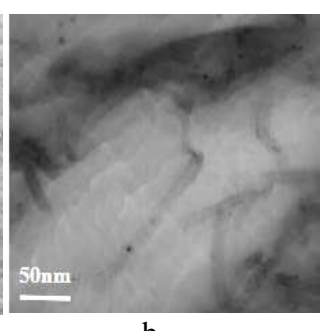

b
Fig. 2. TEM of MWNTs dispersion in LDPE/MWNTs nanocomposites: a-LDPE/1.0 wt.\% MWNTs; b-LDPE/3.0 wt.\% MWNTs

At low concentration of MWNTs (Fig. $1 \mathrm{a}$ and b), some carbon nanotubes are pulled off; some have been pulled out from the LDPE. Due to MWNTs high conductivity, the exposed ends of MWNTs inserting in LDPE display as bright dots; on the other hand, there are some holes resulting from MWNTs pulling out from
LDPE; whatever both the bright dots and the holes are well-distributed, which means a uniform distribution of MWNTs, and no apparent MWNTs aggregations are found in SEM. That MWNTs' one ends still firmly inserting in LDPE matrix means that there exists a stronger interfacial interaction between carbon nanotubes and LDPE, which facilitates the effective transfer of stress in the interfaces from LDPE to MWNTs.

Fig. $2 \mathrm{a}$ and $\mathrm{b}$ display TEM of the ultrathin section of LDPE/1.0 wt.\% MWNTs and LDPE/3.0 wt.\% MWNTs nanocomposites, respectively. It is clear that, in LDPE/1.0 wt.\% MWNTs nanocomposites, as shown in Fig. 2 a, MWNTs are observed the uniform dispersion and the fully extended state in the LDPE matrix; however, there are some aggregations of MWNTs in LDPE/3.0 wt.\% MWNTs nanocomposites. Meanwhile, MWNTS also present the curved shape or are closely interwoven each other due to flexibility of MWNTs (Fig. 2 b), those phenomena explain the MWNTs' nonuniform distribution at high concentration of MWNTs. These observations are in accord with that of SEM. Compared to theoretical reinforcement of MWNTs' straight inclusion, the phenomenon of MWNTs knotting or curvature can obviously decrease the structural reinforcement of MWNTs on LDPE/MWNTs [14]. That most single MWNTs are uniformly dispersed in LDPE (Fig. 2 a) is a key factor for the reinforcement effect of MWNTs in LDPE/MWNTs nanocomposites and has a practical significance. Fig. 1 a, b and Fig. 2 a indicate that, at low concentration of MWNTs, they disperse more evenly.
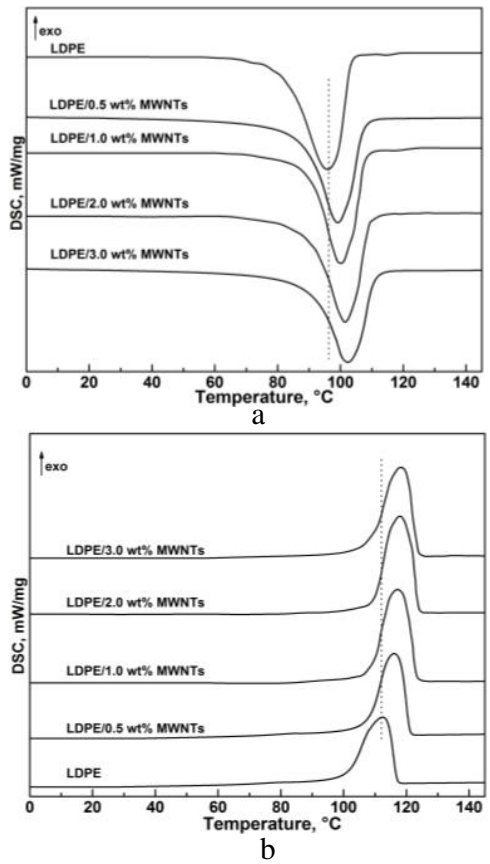

Fig. 3. DSC of LDPE and LDPE/MWNTs nanocomposites: $a$-first cooling; $b$ - second heating

LDPE is a kind of a semi-crystalline polymer. It is necessary to investigate the influence of MWNTs on LDPE crystallization behavior. DSC data for LDPE and LDPE/MWNTs nanocomposites are presented in Fig. 3. The cooling thermograms show that the crystallization temperature $\left(T_{c}\right)$ of LDPE increases with addition of MWNTs (Fig. 3 a). For example, $T_{c}$ of pure LDPE is $96^{\circ} \mathrm{C}$; 
$T_{c}$ of LDPE/0.5 wt.\% MWNTs and LDPE/3.0 wt.\% MWNTs increase to $99{ }^{\circ} \mathrm{C}$ and $102{ }^{\circ} \mathrm{C}$, respectively. Those indicate MWNTs could play a role of a nucleating agent and promote LDPE crystallization; while higher MWNTs contents cannot lead to a significant increase in $T_{c}$. The limited increase of $T_{c}$ in the other polymer-based nanocomposite has been observed. Since there are enough MWNTs, they can provide a sufficient surface area. Consequently, in comparison with a crystal nucleation, the crystal growth is the decisive step in the crystal crystallization rate [15].

The changed trend of melting temperature $\left(T_{m}\right)$ is similar to that of $T_{c}$ in LDPE/MWNTs nanocomposites. However, the increase of $T_{m}$ does not result from the increase of $T_{c}$. HDPE/CNT nanocomposites has been reported the same situation, which ascribes to an improved thermodynamic stability of HDPE crystals in the nanocomposites compared with pure HDPE [15].

Table 1. DSC of LDPE and LDPE/MWNTs nanocomposites

\begin{tabular}{|c|c|c|c|c|c|}
\hline Sample & $\begin{array}{c}T_{c}, \\
{ }^{\circ} \mathrm{C}\end{array}$ & $\begin{array}{c}T_{m}, \\
{ }^{\circ} \mathrm{C}\end{array}$ & $\begin{array}{c}\Delta H_{c}, \\
\mathrm{~J} / \mathrm{g}\end{array}$ & $\begin{array}{c}\Delta H_{m}, \\
\mathrm{~J} / \mathrm{g}\end{array}$ & $\begin{array}{c}X, \\
\%\end{array}$ \\
\hline LDPE & 96 & 112 & 75.6 & 138.4 & 48 \\
\hline LDPE/0.5 wt.\% MWNTs & 99 & 116 & 74.3 & 145.2 & 50 \\
\hline LDPE/1.0 wt.\% MWNTs & 100 & 117 & 73.1 & 147.8 & 51 \\
\hline LDPE/2.0 wt.\% MWNTs & 101 & 117 & 71.6 & 150.5 & 52 \\
\hline LDPE/3.0 wt.\% MWNTs & 102 & 118 & 70.3 & 153.7 & 53 \\
\hline
\end{tabular}

The enthalpies of crystallization and melting is calculated from the thermograms and shown in Table 1. The enthalpy of crystallization $\left(\Delta H_{c}\right)$ decreases as the MWNTs content increases, while the enthalpy of melting $\left(\Delta H_{m}\right)$ increases with increasing of the MWNTs. In the case of LDPE, the crystallization enthalpy $\left(\Delta H_{c}\right)$ is around $75.6 \mathrm{~J} \mathrm{~g}^{-1}$; the values of $\Delta H_{c}$ decreases in the nanocomposites as compared with that of LDPE; the values of $\Delta H_{c}$ are around $74.3 \mathrm{~J} \mathrm{~g}^{-1}$ for LDPE/ $0.5 \mathrm{wt} . \%$ MWNTs, 73.1 $\mathrm{J} \mathrm{g}^{-1}$ for LDPE/1.0 wt.\% MWNTs, $71.6 \mathrm{~J} \mathrm{~g}^{-1}$ for LDPE/2.0 wt.\% MWNTs, and $70.3 \mathrm{~J} \mathrm{~g}^{-1}$ for LDPE/3.0 wt.\% MWNTs nanocomposite, respectively. However, in comparison with LDPE, $\Delta H_{m}$ increases slightly in the nanocomposites; they are around $145.2 \mathrm{~J} \mathrm{~g}^{-1}$ for LDPE/0.5 wt.\% MWNTs, $147.8 \mathrm{~J} \mathrm{~g}^{-1}$ for LDPE/1.0 wt.\% MWNTs, $150.5 \mathrm{~J} \mathrm{~g}^{-1}$ for LDPE/2.0 wt.\% MWNTs, and $153.7 \mathrm{~J} \mathrm{~g}^{-1}$ for LDPE/3.0 wt.\% MWNTs nanocomposite, respectively.

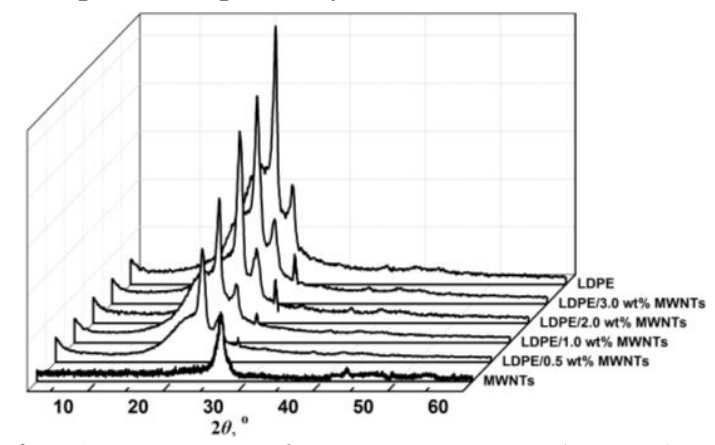

Fig. 4. WAXD patterns of MWNTs, LEPE and LDPE/MWNTs nanocomposites

From the above analysis, the conclusion can be drawn that the incorporation of MWNTs enhances the nonisothermal melt crystallization of LDPE matrix significantly. Table 1 also shows the crystallinity of LDPE/MWNTs nanocomposites with various MWNTs contents. With the increase of MWNTs content, the crystallinity of LDPE/MWNTs nanocomposite rises, which arises from the nucleation effects of MWNTs fillers for crystallization; that is, the existence of MWNTs induces LDPE crystallization, therefore increasing its crystallinity.

It is worth noting whether the introduction of MWNTs affects the crystal structure of LDPE. The WXRD patterns of MWNTs, pure LDPE, and LDPE/MWNTs nanocomposites are presented in Fig. 4. All samples were crystallized at $25^{\circ} \mathrm{C}$ for a day.

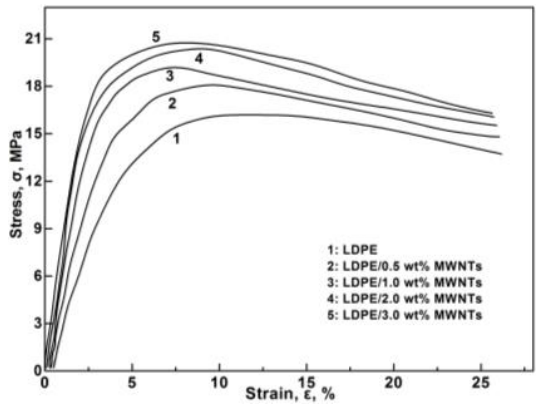

Fig. 5. Stress-strain curves for LDPE and LDPE/MWNTs nanocomposites

The diffraction peaks at $26.4^{\circ}$ and $43.2^{\circ}$ observed in the diffraction of MWNTs can be attributed to the hexagonal graphite structures (002) and (100) [16]. In LDPE and its nanocomposites, the two distinct reflection peaks in the diffractions are appeared at $2 \theta$ of $21.4^{\circ}$ and $23.7^{\circ}$; they correspond to the (110) and (200) lattice planes of orthorhombic crystalline form of LDPE, respectively. These results indicate that LDPE and its nanocomposites exhibit same crystal structure and crystal cell types. In other words, in LDPE/MWNTs nanocomposites, LDPE maintains the original crystal structure in the procedure of blending, and therefore, there is no chemical change at all; the amorphous phase is shown near $2 \theta$ of $19.8^{\circ}$ as a broad peak [17]; meanwhile, the diffraction of MWNTs at $26.4^{\circ}$ can be observed in LDPE/MWNTs nanocomposites; a very interesting is that the strength of amorphous phase of pure LDPE is stronger than that of LDPE/MWNTs nanocomposites. These indicate the size of MWNTs introduced has an important influence on the LDPE crystal. MWNTs could induce LDPE to nucleate during its crystallization process, which can increase the number of LDPE crystal.

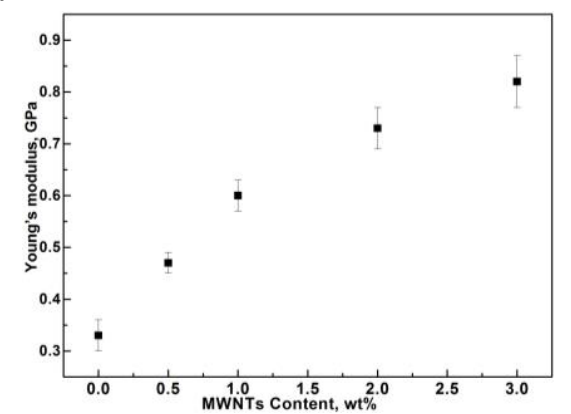

Fig. 6. Young's moduli of LDPE/MWNTs nanocomposites with various MWNTs content

Therefore, with the increase of MWNTs content, in LDPE/MWNTs nanocomposites, the strength of 
amorphous LDPE declines slightly in Fig. 4. MWNTs could clearly enhance the mechanical properties of a polymer. The stress $(\sigma)$-strain $(\varepsilon)$ relation of LDPE/MWNTs nanocomposites is shown in Fig. 5. The strain $(\varepsilon)$ represents an amount of deformation under the action of stress $(\sigma)$, while $\sigma$ is force per area, i.e., the force divided by the cross-sectional area of the unloaded sample. The maximum tensile strength, $\varepsilon_{\max }$, is around $16.4 \mathrm{MPa}$ for LDPE, $18.1 \mathrm{MPa}$ for LDPE/0.5 wt.\% MWNTs, 19.2 MPa for LDPE/1.0 wt.\% MWNTs, 20.4 MPa for LDPE/2.0 wt.\% MWNTs, and $20.9 \mathrm{MPa}$ for LDPE/3.0 wt.\% MWNTs nanocomposite, respectively. Meanwhile, the Young's modulus is around $0.33 \mathrm{GPa}$ for LDPE, $0.47 \mathrm{GPa}$ for LDPE/0.5 wt.\% MWNTs, 0.60 GPa for LDPE/1.0 wt.\% MWNTs, $0.73 \mathrm{GPa}$ for LDPE/2.0 wt.\% MWNTs, and $0.82 \mathrm{GPa}$ for LDPE/3.0 wt.\% MWNTs nanocomposite, respectively, as shown in Fig. 6. Young's modulus of LDPE/MWNTs rises with the increases of MWNTs content. The increase in Young's modulus (148\%) for LDPE/3.0 wt.\% MWNTs nanocomposite, in comparison with pure LDPE, indicates that an effective reinforcement of the MWNTs; it suggests that the significant property enhancement could be attributed to highly dispersed and well aligned MWNTs and/or good adhesion between MWNTs and polymeric matrix.

\section{CONCLUSIONS}

LDPE/MWNTs nanocomposites were manufactured by screw extrusion and injection moulding with $0.5 \%$, $1 \%, 2 \%$ and $3 \%$ (wt.\%) MWNTs content. The results indicate that, for low content of MWNTs, they are uniformly dispersed in LDPE; MWNTs can play a role of a nucleating agent and promote LDPE crystallization; the melting temperature and crystallinity of LDPE/MWNTs increases with the increase of MWNTs content; LDPE crystal maintains the original structure in the procedure of blending with MWNTs; the tensile strength and Young's modulus of LDPE/MWNTs rise with the increases of MWNTs content. The increases in tensile strength and Young's modulus for LDPE/3.0 wt.\% MWNTs nanocomposite can be up to $27 \%$ and $148 \%$, respectively, in comparison with LDPE. It is expected that the prepared LDPE/MWNTs nanocomposites can meet the needs of packaging, transportation and protection, and so on.

\section{REFERENCES}

1. Chehata, N., Ltaief, A., Bkakri, R., Bouazizi, A., Beyou, E. Conducting Polymer Functionalized Multi-walled Carbon Nanotubes Nanocomposites: Optical Properties and Morphological Characteristics Materials Letters 121 2014: pp. 227-230.

2. Xu, W., Ding, Y., Jiang, S., Zhu, J., Ye, W., Shen, Y., Hou, H. Mechanical Flexible PI/MWCNTs Nanocomposites with High Dielectric Permittivity by Electrospinning European Polymer Journal 59 2014: pp. 129-135.

3. Chafidz, A., Kaavessina, M., Al-Zahrani, S., Ali, I. Multiwall Carbon Nanotubes Filled Polypropylene Nanocomposites: Rheological and Electrical Properties Polymer Engineering \& Science 54 (5) 2014: pp. 1134-1143.

4. Kausar, A., Hussain, S.T. Azo-polymer Based Hybrids Reinforced with Carbon Nanotubes and Silver Nanoparticles: Solution and Melt Processing International Journal of
Polymeric Materials and Polymeric Biomaterials

2014: pp. 207-212.

https://doi.org/10.1080/00914037.2013.830248

5. Elksnite, I., Merijs-Meri, R., Reinholds, I., Kalkis, V., Zicans, J., Kalnins, M. Thermal Analysis, Mechanical and Rheological Behaviour of Melt Manufactured Polyethylene/Liquid Crystal Polymer Blends Materials Science $17(2) \quad$ 2011: pp. $145-149$. https://doi.org/10.5755/j01.ms.17.2.483

6. Chen, Z., Liu, Y., Yao, C., Yang, G. Preparation and Characterization of Poly (trimethylene terephthalate)-Poly (ethylene oxide terephthalate) Segmented Copolymer/Multiwalled Carbon Nanotubes Composites by In Situ Polymerization Polymer Engineering \& Science 53 (5) 2013: pp. 914-922.

7. Cadek, M., Coleman, J.N., Ryan, K.P., Nicolosi, V., Bister, G., Fonseca, A., Nagy, J.B., Szostak, K., Béguin, F., Blau, W.J. Reinforcement of Polymers with Carbon Nanotubes: The Role of Nanotube Surface Area Nano Letters 4 (2) 2004: pp. 353-356.

8. Rouba, N., Sadoun, T., Boutagrabet, N., Kerrouche, D., Zadi, S., Mimi, N. Thermo-Oxidation and Biodegradation Study of Low-Density Polyethylene/Starch Films by IR Spectroscopy Iranian Journal of Chemistry \& Chemical Engineering 34 (4) 2015: pp. 69-78.

9. Xiao, K.Q., Zhang, L.C., Zarudi, I. Mechanical and Rheological Properties of Carbon Nanotube-Reinforced Polyethylene Composites Composites Science and Technology 67(2) 2007: pp. 177-182. https://doi.org/10.1016/j.compscitech.2006.07.027

10. Xie, N., Jiao, Q., Zang, C., Wang, C., Liu, Y. Study on Dispersion and Electrical Property of Multi-Walled Carbon Nanotubes/Low-Density Polyethylene Nanocomposites Materials \& Design 31 (4) 2010: pp. 1676-1683.

11. Fetecau, C., Stan, F., Dobrea, D., Birsan, D.C. Influence of Injection Molding Parameters on Mechanical Properties of Low Density Polyethylene Filled with Multiwalled Carbon Nanotubes In ASME 2011 International Mechanical Engineering Congress and Exposition American Society of Mechanical Engineers 2011: pp. 599-605.

12. Dubey, K.A., Bhardwaj, Y.K., Chaudhari, C.V. Sabharwal, S. LDPE/EVA/PCR/MWNT Nanocomposites: Radiation Crosslinking and Physicomechanical Characteristics Polymer Composites 32 (5) 2011: pp. 737-746. https://doi.org/10.1002/pc.21092

13. Oral, E., Beckos, C.G., Ghali, B.W., Lozynsky, A.J., Muratoglu, O.K. Effect of Crosslink Density on the High Pressure Crystallization of UHMWPE Journal of Biomedical Materials Research, Part B: Applied Biomaterials 90 (2) 2009: pp. $720-729$. https://doi.org/10.1002/jbm.b.31340

14. Fisher, F.T., Bradshaw, R.D., Brinson, L.C. Effects of Nanotube Waviness on the Modulus of Nanotube-Reinforced Polymers Applied Physics Letters $80(24)$ 2002: pp. $4647-4649$. https://doi.org/10.1063/1.1487900

15. Grady, B.P., Pompeo, F., Shambaugh, R.L., Resasco, D.E. Nucleation of Polypropylene Crystallization by Single-Walled Carbon Nanotubes Journal of Physical Chemistry B 106 (23) 2002: pp. $5852-5858$. https://doi.org/10.1021/jp014622y

16. Li, W., Liang, C., Zhou, W., Qiu, J., Zhou, Z., Sun, G., Xin, Q. Preparation and Characterization of Multiwalled Carbon Nanotube-supported Platinum for Cathode Catalysts of Direct Methanol Fuel Cells Journal of Physical Chemistry B 107 (26) 2003: pp. 6292-6299.

17. Du, W., Zhong, W., Lin, Y., Shen, L., Du, Q. Space Charge Distribution and Crystalline Structure in Polyethylene Blended with EVOH European Polymer Journal 40 (8) 2004: pp. 1987-1995. https://doi.org/10.1016/j.eurpolymj.2004.04.003 